Bull. Korean Math. Soc. 50 (2013), No. 3, pp. 1029-1040

http://dx.doi.org/10.4134/BKMS.2013.50.3.1029

\title{
CHOW STABILITY OF CANONICAL GENUS 4 CURVES
}

\author{
HosUnG KIM
}

ABstract. In this paper, we give sufficient conditions on a canonical genus 4 curve for it to be Chow (semi)stable.

\section{Introduction}

A Deligne-Mumford stable curve is a complete connected curve $C$ having ample dualising sheaf $\omega_{C}$ and admitting only nodes as singularities. An $n$ canonical curve $C \subset \mathbb{P}^{N}$ is a Deligne-Mumford stable curve of arithmetic genus $g$ embedded by the complete linear system $\left|\omega_{C}^{\otimes n}\right|$ where $N=(2 n-1)(g-1)-1$ if $n \geq 2$, and $N=g-1$ if $n=1$.

Let Chow ${ }_{g, n}$ be the closure of the locus of the Chow forms of $n$-canonical curves of arithmetic genus $g$ in the Chow variety of algebraic cycles of dimension 1 and degree $2 g-2$ in $\mathbb{P}^{N}$. The natural action of $\mathrm{SL}_{N+1}$ on $\mathbb{P}^{N}$ induces an action on Chow $_{g, n}$. Denote the corresponding GIT (Geometric Invariant Theory) quotient space by $\mathrm{Chow}_{g, n} / / \mathrm{SL}_{\mathrm{N}+1}$. To understand this quotient space as a parameter space of curves with some geometric properties, we need to find Chow stability conditions.

Mumford showed that, for $n \geq 5$ and $g \geq 2$, the Chow stable curves are precisely Deligne-Mumford stable curves and there is no strictly Chow semistable curve (cf. [14]). This implies that the quotient space is precisely the moduli space of Deligne-Mumford stable curves $\bar{M}_{4}$.

The cases when $n=3$ and $g \geq 3$ were concerned by Schubert in [16]. He proved that a 3 -canonical curve of genus $g \geq 3$ is Chow stable if and only if it is pseudo-stable and also showed that there is no strictly Chow semistable curve, and thus the quotient space is the moduli space of pseudo-stable curves $\bar{M}_{g}^{p s}$. A pseudo-stable curve is a complete connected curve $C$ satisfying the following properties.

- $\omega_{C}$ is ample,

- it admits at worst nodes and ordinary cusps as singularities, and

Received June 19, 2012; Revised November 9, 2012

2010 Mathematics Subject Classification. Primary 14D22.

Key words and phrases. moduli of curves, chow stability.

This work was supported by National Researcher Program 2010-0020413 of NRF and MEST.

(C)2013 The Korean Mathematical Society 
- it has no elliptic components meeting the rest at one point.

Hyeon and Lee proved that, when $n=3$ and $g=2$, the pseudo-stable curves are indeed Chow semistable and completely classified the strictly Chow semistable points in [10]. They also concerned the case $n=2$ and $g=3$. Hassett and Hyeon studied for the case when $n=2$ and $g \geq 4$ in [8] and the cases when $n=4$ and general $g$ were studied by Hyeon and Morrison in [12].

The purpose of this paper is to study the cases when $n=1$ and $g=4$. More precisely, we want to give sufficient conditions on a canonical genus 4 curve for it to be Chow stable or semistable. To do this, we use the Hilbert-Mumford criterion (cf. Theorem 2.2). Our main results are presented in Section 3.2. We show that any irreducible curve in Chow $_{4,1}$ with mild singularities is Chow stable (cf. Theorem 3.8). For reducible curves, we prove that a general curve in Chow 4,1 with two irreducible components is Chow stable except when it is a union of two elliptic curves meeting at three points (cf. Theorems 3.10 and $3.11)$.

After appearing the preliminary version of this paper, Casalaina-Martin, Jensen, and Laza (cf. [2], Theorem 3.1) classified Chow stable and semistable points in Chow 4,1 by using the GIT analysis for cubic threefolds. Our results are partial but we make a direct computation of the stability conditions on Chow $_{4,1}$.

Throughout this paper, we use the following notations and conventions.

- We work over an algebraically closed field $k$ of characteristic zero.

- A curve is a connected, complete scheme of pure dimension 1.

- For a curve $C$, the genus $g(C)$ of $C$ is its arithmetic genus and we write $\omega_{C}$ for its dualising sheaf.

- We say that a point $p \in C$ is a singular point of type $A_{n}$ if

$$
\hat{\mathcal{O}}_{C, p} \simeq k[[x, y]] /\left(y^{2}-x^{n+1}\right) .
$$

In particular, a node (resp. ordinary cusp) is a singular point of type $A_{1}$ (resp. $A_{2}$ ).

- For a polynomial $P(m)$ of degree $n$ in $m$, we denote by n.l.c. $P(m)$ for the coefficient of $\frac{1}{n !} m^{n}$ in $P(m)$.

\section{Chow stability and canonical embedding}

In this section, we review some basic facts for Chow stability.

\subsection{Chow stability}

A weighted flag $F$ of $\mathbb{P}^{n}$ consists of a choice of coordinates $X_{0}, \ldots, X_{n}$ of $\mathbb{P}^{n}$ and a sequence of integers $r_{0} \geq \cdots \geq r_{n}=0$.

Let $F$ be a weighted flag of $\mathbb{P}^{n}$ as above and $X$ be a variety in $\mathbb{P}^{n}$ of dimension $r$. Let $\alpha: \tilde{X} \rightarrow X$ be a proper birational morphism. Let us define an ideal sheaf $\mathcal{I}(X)$ of $\mathcal{O}_{\tilde{X} \times \mathbb{A}^{1}}$ by

$\mathcal{I}(X) \cdot\left[\alpha^{*} \mathcal{O}_{X}(1) \otimes \mathcal{O}_{\mathbb{A}^{1}}\right]=$ the subsheaf generated by $t^{r_{i}} X_{i}, i=1, \ldots, n$. 
It is well known that $\chi\left(\mathcal{O}_{\tilde{X} \times \mathbb{A}^{1}}(m) / \mathcal{I}(X)^{m} \mathcal{O}_{\tilde{X} \times \mathbb{A}^{1}}(m)\right)$ is a polynomial of degree $r+1$ for $m \gg 0$ (cf. [14], Proposition 2.1). Define

$$
e_{F}(X):=\text { n.l.c. } \chi\left(\mathcal{O}_{\tilde{X} \times \mathbb{A}^{1}}(m) / \mathcal{I}(X)^{m} \mathcal{O}_{\tilde{X} \times \mathbb{A}^{1}}(m)\right) .
$$

Lemma 5.6 in [14] shows that $e_{F}(X)$ does not depend on $\alpha$.

For a Chow cycle $X=\sum a_{i} Y_{i}$ where $Y_{i}$ are subvarieties of $\mathbb{P}^{n}$ of dimension $r$ and $a_{i}$ are nonnegative integer, define

$$
e_{F}(X):=\sum a_{i} e_{F}\left(Y_{i}\right)
$$

Definition 2.1. The natural action of $S L_{n+1}$ on $\mathbb{P}^{n}$ induces an action on the Chow variety of $\mathbb{P}^{n}$. We say that a Chow cycle $X$ in $\mathbb{P}^{n}$ is Chow stable (resp. semistable, unstable) if its Chow from is GIT stable (resp. semistable, unstable) under the action of $S L_{n+1}$ on the Chow variety of $\mathbb{P}^{n}$.

The following theorem is the Hilbert-Mumford criterion which is very useful to determine GIT stability.

Theorem 2.2 ([15], Theorem 2.1). Let $X$ be a Chow cycle of dimension $r$ in $\mathbb{P}^{n}$. Then $X$ is Chow semistable (resp. Chow stable) if and only if

$$
e_{F}(X)-\frac{r+1}{n+1} \operatorname{deg} X \sum r_{i} \leq 0 \quad(\text { resp. }<0)
$$

for any weighted flag $F$ of $\mathbb{P}^{n}$.

\subsection{Criterions for Chow stability}

We now review some methods for determining Chow stability. For more detail, we refer to $[14,15,16]$.

Let $L_{i} \subset \mathbb{P}^{n}$ be the linear subspace defined by $X_{i}=\cdots=X_{n}=0$ and let $P_{L_{i}}: \mathbb{P}^{n}-L_{i} \rightarrow \mathbb{P}^{n-i}$ be the natural projection along $L_{i}$.

Definition 2.3. Let $C \subset \mathbb{P}^{n}$ be an irreducible reduced curve in $\mathbb{P}^{n}$ with $C \not \subset$ $L_{i}$. Let $\alpha_{L_{i}}: \tilde{C} \rightarrow \mathbb{P}^{n-i}$ be the morphism extending the composition of $P_{L_{i}}$ and the normalization $\alpha: \tilde{C} \rightarrow C$. Define

$$
\operatorname{deg} P_{L_{i}}(C):=\left\{\begin{array}{cl}
\left(\operatorname{deg} \alpha_{L_{i}}\right)\left(\operatorname{deg} \alpha_{L_{i}}(\tilde{C})\right) & \text { if } \alpha_{L_{i}}(\tilde{C}) \text { is a curve } \\
0 & \text { otherwise }
\end{array}\right.
$$

and

$$
e_{i}=e_{i}^{F}(C):=\operatorname{deg} C-\operatorname{deg} P_{L_{i}}(C) .
$$

For a Chow cycle $C=\sum a_{j} C_{j}$ where $C_{j}$ is a 1 dimensional subvariety of $\mathbb{P}^{n}$ and $a_{i}$ are nonnegative integer, assume that $C_{j} \not \subset L_{i}$ for all $j$. Define

$$
e_{i}=e_{i}^{F}(C):=\sum a_{j} e_{i}^{F}\left(C_{j}\right) .
$$

From the definition, $e_{0}=0$ and $e_{n}=\operatorname{deg} C$ if $C \not \subset L_{n}$. 
Proposition 2.4 ([14], Corollary 4.11). Let $C \subset \mathbb{P}^{n}$ be a curve such that each irreducible component of $C$ does not contained in $L_{n}$. Then, for any sequence $0=s_{0}<\cdots<s_{l}=n$, it is satisfied that

$$
e_{F}(C) \leq \sum_{i=0}^{l-1}\left(r_{s_{i}}-r_{s_{i+1}}\right)\left(e_{s_{i}}+e_{s_{i+1}}\right)
$$

Let $C$ be an irreducible reduced curve in $\mathbb{P}^{n}$ and let $\alpha: \tilde{C} \rightarrow C$ be the normalization of $C$. Pick a point $p$ in $\tilde{C}$ and let $s$ and $t$ be generators of the maximal ideals of $\mathcal{O}_{\tilde{C}, p}$ and $\mathcal{O}_{\mathbb{A}^{1}, 0}$, respectively. For the natural valuation $v_{p}$ on $\mathcal{O}_{\tilde{C}, p}$, set $\operatorname{ord}_{p} X_{i}:=v_{p}\left(\alpha^{*} X_{i}\right)$. Recall that $\mathcal{I}(C)$ be the ideal sheaf of $\mathcal{O}_{\tilde{C} \times \mathbb{A}^{1}}$ defined by

$\mathcal{I}(C) \cdot\left[\alpha^{*} \mathcal{O}_{C}(1) \otimes \mathcal{O}_{\mathbb{A}^{1}}\right]=$ the subsheaf generated by $t^{r_{i}} \alpha^{*} X_{i}, i=1, \ldots, n$.

For each $p \in \tilde{C}, \mathcal{I}(C)_{p \times\{0\}} \subset \mathcal{O}_{\tilde{C} \times \mathbb{A}^{1}, p \times\{0\}}$ is generated by

$$
t^{r_{0}} s^{\operatorname{ord}_{p} X_{0}}, t^{r_{1}} s^{\operatorname{ord}_{p} X_{1}}, \ldots, t^{r_{n}} s^{\operatorname{ord}_{p} X_{n}},
$$

where $s^{\operatorname{ord}_{p} X_{i}}=0$ if $\operatorname{ord}_{p} X_{i}=\infty$. Let us use the notation

$$
\mathcal{I}(C)_{p \times\{0\}}=\left(t^{r_{0}} s^{\operatorname{ord}_{p} X_{0}}, t^{r_{1}} s^{\operatorname{ord}_{p} X_{1}}, \ldots, t^{r_{n}} s^{\operatorname{ord}_{p} X_{n}}\right) .
$$

Definition 2.5. In the situation above, suppose that there is an $i$ with $r_{i}=0$ and $C \not \subset\left(X_{i}=0\right)$. For each point $p$ in $\tilde{C}$, we define

$$
e_{F}(\tilde{C})_{p}:=\text { n.l.c. } \operatorname{dim}_{k}\left(\mathcal{O}_{\tilde{C} \times \mathbb{A}^{1}, p \times\{0\}} / \mathcal{I}(C)_{p \times\{0\}}^{m}\right) .
$$

Remark 2.6. In the setting of Definition 2.5, the quotient sheaf $\mathcal{O}_{\tilde{C} \times \mathbb{A}^{1}} / \mathcal{I}(C)$ is supported at the points over $C \cap L_{n}$ because $r_{n}=0$. Therefore

$$
\begin{aligned}
e_{F}(C) & =\text { n.l.c. } \chi\left(\mathcal{O}_{\tilde{C} \times \mathbb{A}^{1}}(m) / \mathcal{I}(C)^{m} \mathcal{O}_{\tilde{C} \times \mathbb{A}^{1}}(m)\right) \\
& =\sum_{\alpha(p) \in L_{n}} \operatorname{dim}_{k}\left(\mathcal{O}_{\tilde{C} \times \mathbb{A}^{1}, p \times\{0\}} / \mathcal{I}(C)_{p \times\{0\}}^{m}\right) \\
& =\sum_{\alpha(p) \in L_{n}} e_{F}(\tilde{C})_{p}
\end{aligned}
$$

Lemma 2.7 ([16], Lemma 1.4). In the situation of Definition 2.5, set $v_{i}:=$ $\operatorname{ord}_{p} X_{i}$. If $v_{i}+r_{i} \geq a$ for all $i=0, \ldots, n$, then $e_{F}(\tilde{C})_{p} \geq a^{2}$.

\subsection{Canonical curves}

Definition 2.8. We say that a curve $C$ is honestly hyperelliptic if there is a morphism $C \rightarrow \mathbb{P}^{1}$ of degree 2 , and is honestly non-hyperelliptic if it is not honestly hyperelliptic.

A Gorenstein curve is a curve $C$ with $\omega_{C} \cong \mathcal{O}_{C}\left(K_{C}\right)$ for a Cartier divisor $K_{C}$. A generically Gorenstein curve is a curve $C$ such that $\omega_{C}$ is locally isomorphic to $\mathcal{O}_{C}$ outside a finite set. 
Theorem 2.9 ([1], Theorem 3.6). Let $C$ be a numerically 3-connected Gorenstein curve. That is, for any generically Gorenstein strict subcurve $D \subset C$,

$$
\operatorname{deg} \mathcal{O}_{D}\left(K_{C}\right)-\operatorname{deg} \omega_{D} \geq 3 .
$$

Then either $C$ is honestly hyperelliptic or $K_{C}$ is very ample.

If $C$ is a numerically 3 -connected curve admitting nodal singularities only, then Theorem 2.9 implies that any irreducible component of $C$ has at least three intersection points with the union of the other components.

Definition 2.10. A canonical curve is a numerically 3 -connected honestly nonhyperelliptic Gorenstein genus $g$ curve $C \subset \mathbb{P}^{g-1}$ whose embedding is given by $\left|\omega_{C}\right|$.

We remark that any canonical curve $C \subset \mathbb{P}^{g-1}$ is a nondegenerate curve of degree $2 g-2$.

\section{Canonical curves of genus four}

From now on, $F$ is a weighted flag of $\mathbb{P}^{3}$ associated with coordinates $X_{0}, \ldots$, $X_{3}$ and weights $r_{0} \geq \cdots \geq r_{3}=0$, and $L_{i}$ is the linear subspace of $\mathbb{P}^{3}$ defined by $X_{i}=\cdots=X_{3}=0$.

Note that any canonical genus 4 curve in $\mathbb{P}^{3}$ has degree 6 . Thus applying Theorem 2.2 we get that a canonical genus 4 curve $C \subset \mathbb{P}^{3}$ is Chow stable (resp. semistable) if and only if

$$
e_{F}(C)<(\text { resp. } \leq) 3 \sum r_{i}
$$

for any weighted flag $F$.

\subsection{Upper bounds of $e_{F}(C)$}

In this subsection, we gather some preliminary results which will be used to give upper bounds of $e_{F}(C)$ in the next subsection.

Lemma 3.1. Let $C \subset \mathbb{P}^{3}$ be a curve of degree $d$, and let $e_{i}$ be the same as that in Definition 2.3. Assume that each irreducible component of $C$ does not contained in $L_{n}$. Then

$$
e_{F}(C) \leq \min \left\{d r_{0}, e_{1} r_{0}+d r_{1}, e_{2} r_{0}+d r_{2}, e_{1} r_{0}+e_{2} r_{1}+\left(d-e_{1}\right) r_{2}\right\} .
$$

Proof. The lemma immediately comes by applying Proposition 2.4 to the sequences $0<3,0<1<3,0<2<3$ and $0<1<2<3$.

Lemma 3.2. Let $R:=k[s, t]$ and $I$ an ideal of $R$.

(1) If $I=\left(t^{a}, s^{b}\right)$ for integers $a, b \geq 1$, then n.l.c. $\operatorname{dim}_{k} R / I^{m}=a b$.

(2) If $I=\left(t^{a}, t^{p} s^{q}, s^{b}\right)$ for integers $a, b, p, q \geq 1$, then

$$
\text { n.l.c. } \operatorname{dim}_{k} R / I^{m} \leq a q+b p \text {. }
$$


Proof. If $I=\left(t^{a}, s^{b}\right)$, then $I^{m}$ is generated by

$$
\left\{t^{a n_{1}+r_{1}} s^{b n_{2}+r_{2}} \mid n_{1}+n_{2} \geq m, 0 \leq r_{1}<a, 0 \leq r_{2}<b\right\} .
$$

Thus the following set of monomials

$$
\left\{s^{b i+k} t^{j} \mid 0 \leq i \leq m-1,0 \leq j \leq a(m-i)-1,0 \leq k \leq b-1\right\}
$$

forms a basis of $R / I^{m}$. Therefore

$$
\operatorname{dim}_{k} R / I^{m}=\sum_{i=0}^{m-1} a(m-i) b=a b\left(m^{2}+m\right) / 2,
$$

which implies (1). Similarly, (2) can be proved by describing the set of the monomials spanning $R / I^{m}$.

Lemma 3.3. Let $C \subset \mathbb{P}^{3}$ be a curve of degree $d$, and assume that each irreducible component of $C$ does not lie in the hyperplane $L_{3}$. Then

$$
e_{F}(C) \leq\left(\sum_{\alpha(p)=L_{1}} \operatorname{ord}_{p} X_{3}\right) r_{0}+\left(\sum_{\alpha(p) \in L_{2}-L_{1}} \operatorname{ord}_{p} X_{3}\right) r_{1}+\left(\sum_{\alpha(p) \notin L_{2}} \operatorname{ord}_{p} X_{3}\right) r_{2} .
$$

Proof. We may assume that $C$ is irreducible and reduced. Let $\alpha: \tilde{C} \rightarrow C$ be the normalization of $C$. Take a point $p$ in $\tilde{C}$ and set $v_{i}=\operatorname{ord}_{p} X_{i}$. Then

$$
\mathcal{I}(C)_{p \times\{0\}}=\left(t^{r_{0}} s^{v_{0}}, t^{r_{1}} s^{v_{1}}, t^{r_{2}} s^{v_{2}}, s^{v_{3}}\right) .
$$

From this, it is induced that

$$
\mathcal{I}(C)_{p \times\{0\}}> \begin{cases}\left(t^{r_{0}}, s^{v_{3}}\right), & \text { for all } p \\ \left(t^{r_{1}}, s^{v_{3}}\right), & \text { if } \alpha(p) \neq L_{1} \\ \left(t^{r_{2}}, s^{v_{3}}\right), & \text { if } \alpha(p) \notin L_{2} .\end{cases}
$$

Applying Lemma 3.1 to these inclusions, we obtain that

$$
e_{F}(\tilde{C})_{p} \leq \begin{cases}r_{0} v_{3}, & \text { for all } p \\ r_{1} v_{3}, & \text { if } \alpha(p) \neq L_{1} \\ r_{2} v_{3}, & \text { if } \alpha(p) \notin L_{2} .\end{cases}
$$

Using the equality $e_{F}(C)=\sum_{p \in \tilde{C}} e_{F}(\tilde{C})_{p}$, the desired inequality can be verified.

Lemma 3.4. Let $C \subset \mathbb{P}^{3}$ be a reduced irreducible curve of degree $d$ and assume that $C \subset L_{3}$ and $C \neq L_{2}$. Then

$$
e_{F}(C) \leq\left(\sum_{\alpha(p)=L_{1}} \operatorname{ord}_{p} X_{3}\right) r_{0}+\left(\sum_{\alpha(p) \neq L_{1}} \operatorname{ord}_{p} X_{i}\right) r_{1}+d r_{2} .
$$

Proof. Let $\alpha: \tilde{C} \rightarrow C$ be the normalization of $C$. Let $F^{\prime}$ be the weighted flag of $L_{3} \cong \mathbb{P}^{2}$ associated with the coordinates $X_{0}^{\prime}:=\left.X_{0}\right|_{L_{3}}, X_{1}^{\prime}:=\left.X_{1}\right|_{L_{3}}$, 
$X_{2}^{\prime}:=\left.X_{2}\right|_{L_{3}}$ and the weights $r_{0}^{\prime}=r_{0}-r_{2} \geq r_{1}^{\prime}=r_{1}-r_{2} \geq r_{2}^{\prime}=0$. From the proof of Theorem 2.9 in [14] it is induced that

$$
e_{F}(C)=e_{F^{\prime}}(C)+2 d r_{2} \text {. }
$$

Take a point $p \in \tilde{C} \cap\left(X_{2}^{\prime}=0\right)$ and set $v_{i}:=\operatorname{ord}_{p} \alpha^{*} X_{i}$. Then

$$
e_{F^{\prime}}(\tilde{C})_{p} \leq \begin{cases}r_{0}^{\prime} v_{2}, & \text { for all } p \\ r_{1}^{\prime} v_{2}, & \text { if } \alpha(p) \neq L_{1} .\end{cases}
$$

The first inequality is given by applying from Lemma 3.2 to the inclusion

$$
\mathcal{I}(C)_{p \times\{0\}}=\left(t^{r_{0}^{\prime}} s^{v_{0}}, t^{r_{1}^{\prime}} s^{v_{1}}, s^{v_{2}}\right)>\left(t^{r_{0}^{\prime}}, s^{v_{2}}\right) .
$$

If $\alpha(p) \neq L_{1}$, then $v_{1}=0$, and hence we get the next inclusion

$$
\mathcal{I}(C)_{p \times\{0\}}>\left(t^{r_{1}^{\prime}}, s^{v_{2}}\right)
$$

which implies the second inequality by Lemma 3.2. From the equality $e_{F^{\prime}}(C)=$ $\sum_{p \in \tilde{C}} e_{F^{\prime}}(\tilde{C})_{p}$, we get the lemma.

Lemma 3.5. If $C \subset \mathbb{P}^{3}$ is equal to $L_{2}$, then $e_{F}(C)=r_{0}+r_{1}$.

Proof. The coordinate ring of $C$ is $R=k\left[X_{0}, X_{1}\right]$. Let $I=\left(X_{0} t^{r_{0}}, X_{1} t^{r_{1}}\right)$. Applying Lemma 1.3 in [16], we get that

$$
e_{F}(C)=\text { n.l.c. } \operatorname{dim}_{k}\left(R[t] / I^{m}\right)_{m} .
$$

Since $I^{m}$ is generated by

$$
\left\{t^{r_{0} i+r_{1} j} X_{0}^{i} X_{1}^{j} \mid i+j=m\right\},
$$

we get that

$$
\begin{aligned}
\operatorname{dim}_{k}\left(R[t] / I^{m}\right)_{m} & =\sum_{i+j=m} r_{0} i+r_{1} j=\sum_{i=0}^{m} r_{0} i+r_{1}(m-i) \\
& =\sum_{i=0}^{m} i\left(r_{0}-r_{1}\right)+m r_{1} \\
& =\frac{m(m+1)}{2}\left(r_{0}-r_{1}\right)+m(m+1) r_{1} \\
& =\frac{r_{0}+r_{1}}{2}\left(m^{2}+m\right)
\end{aligned}
$$

and thus the required equality is obtained.

\subsection{Main results}

Next proposition says that Chow stable curves admit at worst double points.

Proposition 3.6. Let $C \subset \mathbb{P}^{3}$ be a curve of degree 6 . If $C$ admits a singular point of multiplicity $\geq 3$, then it is not Chow stable. Furthermore, if $C$ has a point of multiplicity $\geq 4$, then it is not Chow semistable. 
Proof. Let $p$ be a point of $C$ with multiplicity bigger than or equal to 3 . Take coordinates $X_{0}, \ldots, X_{3}$ so that $X_{1}, X_{2}$, and $X_{3}$ vanish at $p$, and let $r_{0}=1, r_{1}=$ $r_{2}=r_{3}=0$. For the associated weighted flag $F$, it follows that

$$
\mathcal{I}_{p \times\{0\}}(C)=\left(t, m_{p}\right) \mathcal{O}_{C \times \mathbb{A}^{1}, p \times\{0\}}
$$

which is the maximal ideal of $\mathcal{O}_{C \times \mathbb{A}^{1}, p \times\{0\}}$ where $m_{p}$ is the maximal ideal of $\mathcal{O}_{C, p}$. Hence

$$
e_{F}(C)=e_{F}(C)_{p}=\operatorname{mult}_{p \times\{0\}}\left(C \times \mathbb{A}^{1}\right)=\operatorname{mult}_{p} C \geq 3=3 \sum r_{i} .
$$

Furthermore, the last inequality is strict if $\operatorname{mult}_{p} C \geq 4$.

The next proposition will be used in the proof of the following theorems.

Proposition 3.7. Let $C \subset \mathbb{P}^{3}$ be an honestly non-hyperelliptic curve of degree 6 in the sense of Definition 2.8, and assume that each irreducible component of $C$ does not contained in $L_{n}$. Suppose that $e_{1} \leq 2$ and $e_{2} \leq 4$ where $e_{i}$ be the same as that in Definition 2.3. Then $C$ is Chow stable with respect to $F$.

Proof. From Lemma 3.1, it follows that

$$
e_{F}(C) \leq \min \left\{6 r_{0}, 2 r_{0}+6 r_{1}, 4 r_{0}+6 r_{2}, 2 r_{0}+4 r_{1}+4 r_{2}\right\} \text {. }
$$

If the right hand side in the above inequality is greater than or equal to $3 \sum r_{i}$ simultaneously, then it should be satisfied that

$$
6 r_{0}=2 r_{0}+6 r_{1}=4 r_{0}+6 r_{2}=2 r_{0}+4 r_{1}+4 r_{2}=3 \sum r_{i}
$$

which implies that $e_{F}(C) \leq 3 \sum r_{i}$, and the equality $e_{F}(C)=3 \sum r_{i}$ holds only when $r_{0}=3 r, r_{1}=2 r, r_{2}=r$ for some $r \in \mathbb{Z}_{>0}$, and $e_{2}=2, e_{4}=4$.

We now assume that $r_{0}=3 r, r_{1}=2 r$ and $r_{2}=r$ for some $r \in \mathbb{Z}_{>0}$, and $e_{1}=2$ and $e_{4}=4$. If $C$ meets $L_{3}$ at a point not equal to $L_{1}$, then

$$
e_{F}(C) \leq 5 r_{0}+r_{1}=17 r<3 \sum r_{i}
$$

by Lemma 3.3. On the other hand, if $C$ intersects $L_{3}$ only at $L_{1}$, then the restricted projection morphism $\left.P_{L_{2}}\right|_{C \cap\left(\mathbb{P}^{3}-L_{2}\right)}$ extends to a morphism $C \rightarrow$ $\mathbb{P}^{1}$ of degree 2 because $e_{2}=4$ and the assumption that $C \cap L_{3}$ consists of only one point $L_{1}$. This gives a contradiction because $C$ is honestly nonhyperelliptic.

Our next result shows that any irreducible canonical curve admitting only mild singularities is Chow stable.

Theorem 3.8. Let $C \subset \mathbb{P}^{3}$ be an irreducible canonical curve of genus 4 admitting at worst $A_{n}, n \leq 4$, singularities. Then $C$ is Chow stable.

Proof. From the assumptions, it is induced that $C$ admits at most double points, and is nondegenerate. Thus it follows that $e_{1} \leq 2$ and $e_{2} \leq 5$. Via Proposition 3.7, it is enough to show that $e_{2} \neq 5$. Suppose not. The composition of the partial normalization morphism $\tilde{C} \rightarrow C$ at the points in $C \cap L_{2}$ 
and the restricted projection morphism $\left.P_{L_{2}}\right|_{C \cap\left(\mathbb{P}^{3}-L_{2}\right)}$ induces an isomorphism $\tilde{C} \rightarrow \mathbb{P}^{1}$. This shows that $C$ has exactly two double points $P$ and $Q$ of type $A_{3}$ or $A_{4}$, and $L_{2}$ meets $C$ at $P, Q$ and another point. Let us denote by $H$ the plane determined by $L_{2}$ and the tangent line of $C$ at $P$. Then the number of intersection points of $H$ and $C$ is greater than or equal to 7 with multiplicity, a contradiction.

The next theorem deals with double twisted curves in $\mathbb{P}^{3}$ which are the canonical images of smooth hyperelliptic curves of genus 4 .

Theorem 3.9. Let $C \subset \mathbb{P}^{3}$ be a double curve supported on a twisted cubic curve. Then $C$ is Chow semistable but not stable. Moreover, all such curves are identified in Chow $_{4,1} / / \mathrm{SL}_{4}$.

Proof. Let $C=2 C_{1}$ where $C_{1}$ is the twisted cubic curve in $\mathbb{P}^{3}$. Then $e_{1} \leq 1$ and $e_{2} \leq 2$ for $C_{1}$, and thus $e_{F}(C)=2 e_{F}\left(C_{1}\right)$ is less than or equal to $4 r_{0}+6 r_{2}$ and $2 r_{0}+6 r_{1}$ by Lemma 3.1. On the other hand, the two values $4 r_{0}+6 r_{2}$ and $2 r_{0}+6 r_{1}$ cannot be bigger than $3 \sum r_{i}$ simultaneously, which implies that $C$ is Chow semistable.

Take a point $p$ in $C_{1}$, and choose coordinates $X_{0}, \ldots, X_{3}$ so that $X_{1}, X_{2}, X_{3}$ vanish at $p, X_{2}, X_{3}$ vanish to order $\geq 2$ at $p$, and $X_{3}$ vanishes to order $\geq 3$ at $p$. Set $r_{0}=3, r_{1}=2, r_{2}=1, r_{3}=0$. For the corresponding weighted flag $F$, it is obtained that

$$
e_{F}(C)=2 e_{F}\left(C_{1}\right) \geq 2 e_{F}\left(C_{1}\right)_{p} \geq 2 \cdot 9=3 \sum r_{i}
$$

by Lemma 2.7 , and thus $C$ is not Chow stable.

The last statement comes from the fact that any two twisted cubic curves are projective equivalent.

Let $\delta_{i, j} \subset$ Chow $_{4,1}$ be the closure of the locus parametrizing canonical curves consisting of two smooth components meeting at nodes and having genus $i$ and $j$ respectively. Let $C$ be a curve in $\delta_{i, j}$ with two smooth irreducible components $C_{1}$ and $C_{2}$ meeting at $r$ nodes. Then $r \geq 3$ by the remark after Theorem 2.9. Moreover

$$
g(C)=g\left(C_{1}\right)+g\left(C_{2}\right)+r-1=i+j+r-1=4 .
$$

Thus the only nontrivial cases are $\delta_{1,1}, \delta_{2,0}, \delta_{1,0}$ and $\delta_{0,0}$.

Throughout Theorems 3.10 and 3.11 , we will show that a general curve in each $\delta_{i, j}$ is Chow stable except when it belongs to a class in $\delta_{1,1}$.

Theorem 3.10. If $C \subset \mathbb{P}^{3}$ is a general curve in $\delta_{1,1}$, then it is Chow semistable but not Chow stable. Furthermore, all Chow semistable curves in $\delta_{1,1}$ are identified in Chow $_{4,1} / / \mathrm{SL}_{4}$.

Proof. Without loss of generality, we may assume that $C$ is a union of two smooth elliptic curves $C_{1}$ and $C_{2}$ meeting at three nodes denoted by $p_{1}, p_{2}$ and $p_{3}$. Note that each $C_{i}$ is contained in a hyperplane denoted by $H_{i}$, and has degree 3 . 
If $L_{2}$ is not contained in any $H_{i}$, then $e_{1} \leq 2$ and $e_{2} \leq 4$ which implies that $e_{F}(C) \leq 3 \sum r_{i}$ by Proposition 3.7, and thus we may assume that $L_{2} \subset H_{2}$.

If $H_{2}$ is not equal to $L_{3}$, then

$$
e_{F}(C)=e_{F}\left(C_{1}\right)+e_{F}\left(C_{2}\right) \leq \begin{cases}\left(r_{0}+2 r_{1}\right)+\left(r_{0}+2 r_{1}\right), & \text { if } L_{2}=H_{1} \cap H_{2} \\ \left(r_{0}+2 r_{2}\right)+\left(r_{0}+3 r_{1}\right), & \text { if } L_{2} \neq H_{1} \cap H_{2}\end{cases}
$$

which implies that $e_{F}(C) \leq 3 \sum r_{i}$.

Now assume that $H_{2}=L_{3}$. Then it is easy to check that

$e_{F}(C)=e_{F}\left(C_{1}\right)+e_{F}\left(C_{2}\right) \leq \begin{cases}\left(r_{0}+2 r_{1}\right)+\left(r_{0}+2 r_{1}+3 r_{2}\right), & \text { if } L_{2}=H_{1} \cap H_{2} \\ \left(r_{0}+2 r_{2}\right)+\left(r_{0}+2 r_{1}+3 r_{2}\right), & \text { if } L_{2} \neq H_{1} \cap H_{2}\end{cases}$

which yields that $e_{F}(C) \leq 3 \sum r_{i}$. Finally we showed that $C$ is Chow semistable.

Choose coordinates $X_{0}, \ldots, X_{3}$ so that $H_{1}$ and $H_{2}$ are hyperplanes defined by $X_{2}=0$ and $X_{3}=0$ respectively. Set $r_{0}=r_{1}=r$ and $r_{2}=0$. Then for each $i$ it follows that

$$
e_{F}\left(C_{i}\right)=e_{F}\left(C_{i}\right)_{p_{1}}+e_{F}\left(C_{i}\right)_{p_{2}}+e_{F}\left(C_{i}\right)_{p_{3}}=r+r+r=3 r
$$

and thus

$$
e_{F}(C)=e_{F}\left(C_{1}\right)+e_{F}\left(C_{2}\right)=6 r=3 \sum r_{i} .
$$

This shows that $C$ is not Chow stable.

Now it remains to show the last statement of the theorem. Choose coordinates $X_{0}, \ldots, X_{3}$ of $\mathbb{P}^{3}$ so that $C$ is defined by

$$
X_{0} X_{2}^{2}+X_{0} X_{3}^{2}-X_{1}\left(X_{1}-a X_{0}\right)\left(X_{1}-b X_{0}\right)=0 \text { and } X_{2} X_{3}=0,
$$

where $1, a$ and $b$ are distinct where $X_{0}, \ldots, X_{3}$ is a homogeneous coordinates on $\mathbb{P}^{3}$. Note that general curve satisfying the assumptions in the proposition can be defined in this way if we choose suitable coordinates.

Consider the one parameter subgroup $\lambda: \mathbb{G}_{m} \rightarrow \mathrm{GL}_{4}$ defined by

$$
\lambda(t) X_{0}=t X_{0}, \lambda(t) X_{1}=t X_{1}, \lambda(t) X_{2}=X_{2} \text {, and } \lambda(t) X_{3}=X_{3} .
$$

Let $\bar{C}$ be the limit of $C$ as $t \rightarrow \infty$ under the action $\lambda$. Applying the computation in [9], it follows that $\bar{C}$ is given by

$$
X_{1}\left(X_{1}-a X_{0}\right)\left(X_{1}-b X_{0}\right)=0 \text { and } X_{2} X_{3}=0 .
$$

We note that $\bar{C}$ is a union of $\bar{C}_{1}$ and $\bar{C}_{2}$ satisfying

(a) each $\bar{C}_{i}$ is contained in $H_{i}$,

(b) $\bar{C}_{1}=L_{1,1} \cup L_{1,2} \cup L_{1,3}$ and $\bar{C}_{2}=L_{2,1} \cup L_{2,2} \cup L_{2,3}$ where each $L_{i, j}$ is a line,

(c) $L_{i, 1}, L_{i, 2}$ and $L_{i, 3}$ intersect at one point $q_{i}$ for each $i=1,2$, and

(d) $L_{1, j}$ and $L_{2, j}$ meet at a point $p_{j}$.

From Section 11.3 in [4], it is induced that $\bar{C}$ is Chow semistable. Note that any two curves satisfying $(\mathrm{a}) \sim(\mathrm{d})$ are projectively equivalent which yields the last statement in the theorem. 
Theorem 3.11. If $C \subset \mathbb{P}^{3}$ is a general curve in $\delta_{2,0}, \delta_{1,0}$, or $\delta_{0,0}$, then it is Chow stable.

Proof. Without loss of generality, we may assume that $C$ is a canonical curve consisting of two smooth components $C_{1}$ and $C_{2}$ meeting at nodes. It is easy to check that $e_{1} \leq 2$ and $e_{2} \leq 4$ for any weighted flag $F$. Therefore by Proposition 3.7, we can also assume that $C_{2}$ is contained in $L_{3}$.

If $C$ belongs to a class in $\delta_{0,0}$, then $C_{1}$ and $C_{2}$ are twisted cubic curves by Fig. 18 in p. 354 [5], and thus they are nondegenerate, a contradiction.

Assume that $C$ belongs to a class in $\delta_{1,0}$. From Fig. 18 in p. 354 [5], we obtain that $\operatorname{deg} C_{1}=4$ and $\operatorname{deg} C_{2}=2$. We note that the intersection $C_{1} \cap C_{2}$ consists of four distinct nodes of $C$ and $C_{2} \subset L_{3}$. Therefore the points in $C_{1} \cap L_{3}$ are exactly the same as that in $C_{1} \cap C_{2}$. Hence in $C_{1} \cap L_{3}$, there exist at least two points not lying on $L_{2}$, and at least three points not equal to $L_{1}$, which implies that $e_{F}\left(C_{1}\right) \leq r_{0}+r_{1}+2 r_{2}$ by Lemma 3.3. Applying Lemma 3.4 , it is induced that $e_{F}\left(C_{2}\right) \leq 2 r_{0}+2 r_{2}$. Therefore

$$
e_{F}(C)=e_{F}\left(C_{1}\right)+e_{F}\left(C_{2}\right) \leq 3 r_{0}+r_{1}+4 r_{2} \leq 3 \sum r_{i} .
$$

In the last inequality, the equality holds if and only if $r_{1}=r_{2}=0$. In the case when $r_{1}=r_{2}=0$, it is induced that

$$
e_{F}(C)=e_{F}\left(C_{1}\right)+e_{F}\left(C_{2}\right)=e_{F}\left(C_{1}\right)_{p}+e_{F}\left(C_{2}\right)_{p} \leq 2 r<3 \sum r_{i},
$$

where $p$ is the point on which $X_{1}, X_{2}$ and $X_{3}$ vanish.

The cases when $C$ belongs to a class in $\delta_{2,0}$ can be proved by similar arguments.

Acknowledgements. The author would like to thank a referee for valuable comments to modify the original version. This paper is a large part of the author's thesis under Professor Yongnam Lee. The author wishes to thank him for his kind guidance.

\section{References}

[1] F. Catanese, M. Franciosi, K. Hulek, and M. Reid, Embeddings of curves and surfaces, Nagoya Math. J. 154 (1999), 185-220.

[2] S. Casalaina-Martin, D. Jensen, and R. Laza, The geometry of the ball quotient model of the moduli space of genus four curves, In Compact moduli spaces and vector bundles, 107-136, Contemp. Math., 564, Amer. Math. Soc., Providence. RI, 2012.

[3] P. Deligne and D. Mumford, The irreducibility of the space of curves of given genus, Inst. Hautes Études Sci. Publ. Math. No. 36 (1969), 75-109.

[4] I. Dolgachev, Lectures on Invariant Theory, London Mathematical Society Lecture Note Series, 296, Cambridge University Press, Cambridge, 2003.

[5] R. Hartshorne, Algebraic Geometry, Graduate Texts in Mathematics, No. 52, SpringerVerlag, New York-Heidelberg, 1977.

[6] B. Hassett, Local stable reduction of plane curve singularities, J. Reine Angew. Math. 520 (2000), 169-194.

[7] B. Hassett and D. Hyeon, Log canonical models for the moduli space of curves: the first divisorial contraction, Trans. Amer. Math. Soc. 361 (2009), no. 8, 4471-4489. 
[8] L Log minimal program for the moduli space of stable curves: the first flip, Preprint, 2008

[9] B. Hassett, D. Hyeon, and Y. Lee, Stability computation via Grobner basis, J. Korean Math. Soc. 47 (2010), no. 1, 41-62.

[10] D. Hyeon and Y. Lee, Stability of tri-canonical curves of genus two, Math. Ann. 337 (2007), no. 2, 479-488.

[11] Stability of bicanonical curves of genus three, J. Pure Appl. Algebra 213 (2009), no. 10, 1991-2000.

[12] D. Hyeon and I. Morrison, Stability of tails and 4-canonical models, Math. Res. Lett. 17 (2010), no. 4, 721-729.

[13] A. Moriwaki, A sharp slope inequality for general stable fibrations of curves, J. Reine Angew. Math. 480 (1996), 177-195.

[14] D. Mumford, Stability of projective varieties, Enseignement Math. (2) 23 (1977), no. $1-2,39-110$

[15] D. Mumford and J. Fogarty, Geometric Invariant Theory, Second edition. Ergebnisse der Mathematik und ihrer Grenzgebiete, 34, Springer-Verlag, Berlin, 1982.

[16] D. Schubert, A new compactification of the moduli space of curves, Compositio Math. 78 (1991), no. 3, 297-313.

Department of Mathematics

Korea Institute for Advanced Study

SEOUl 130-722, Korea

E-mail address: hosung@sogang.ac.kr 